\title{
DEVELOPMENT AND DEMONSTRATION OF SATELLITE IMAGE SALINITY ANALYZER - A TOOL FOR SALINITY MAPPING
}

\author{
Rekha $\mathbf{S}^{1}$, Jenita $\mathbf{R}^{1}$, Mrunalini $\mathbf{B}^{1}$, Kannan $\mathrm{V}^{2}$ \& Nethaji Mariappan V. $\mathbf{E}^{3}$ \\ ${ }^{1}$ Student, Department of ECE, ${ }^{2} \mathrm{HOD}$, Dept. of VLSI, ${ }^{3}$ Scientist-D, Centre for Remote Sensing and \\ Geoinformatics, Sathyabama University, Rajiv Gandhi Road, Jeppiaar Nagar, Chennai - 600119. \\ *Email: nethajim@gmail.com
}

\begin{abstract}
Soil salinity is caused by natural or human induced process and considered to be a major environmental hazard. Nearly $20 \%$ of all irrigated land in India was salt affected and this proportion tends to increase in spite of considerable efforts dedicated to land reclamation. This requires careful monitoring of the soil salinity status and the variation to the curb degradation trends and secure sustainable land use and management. This study area Nagapattinam district was affected by salinity, the situation became worse after tsunami salinity level has increased that makes portable water un-utilizable and change in cropping pattern even at some places saltpans are available for extracting salt by salt industry. The paper attempts to demonstrate a tool for identifying and mapping salt affected areas and also deriving different levels of soil salinity in order to cope with increasing and sustaining the productivity of crops from remote sensing data using various threshold levels.
\end{abstract}

\section{INTRODUCTION}

Globally, it has been realized that spatial information has become an essential element in the development of a country. Technologies like GIS enable the analysis and management of a variety of data sources for the purpose of a better understanding of environmental processes, sound decision making, a sustainable management of natural resources and disaster management. Consequently the demand on qualitative, accurate timely and

up-to-date spatial information has increase rapidly over the last decades. In developing countries an obstacle to content this demand in the limited availability of reliable is monitoring systems. Earth observation data obtained from satellites can be used as major tool for addressing these structural limitations. But also in industrialized countries remote sensing techniques often represent the only feasible way to monitor a geophysical parameter regularly over long time with an adequate resolution. Nagapattinam a Coastal District of Tamil Nadu, on the eastern coast, Bay of Bengal, 326 K.M, south of the State Capital Chennai was taken as the study area. District lies between Northern Latitude 10.7906 degrees and 79.8428 Degrees Eastern Longitude.

\section{Data Used}

The LANDSAT satellite data of path 142 and row 53 from the websitewww.glcf.umd.edu:8080 /esdi/index.jsp and date of acquisition is September $3^{\text {rd }}$ 2006.

Data contains seven bands out of which band 2, 3 \& 4 corresponding to green, red and NIR (Near Infra Red) bands are downloaded from the USGS website. These data sets were projected with UTM (Universal Transverse Mercator) projection and utilized by the remote sensing software like ERDAS IMAGINE for layer staking. The South Nagapattinam boundary files were overlaid above the image and subsetted for the study area. Preliminary analysis such as histogram equilisation, brightness contrast, linear stretch were attempted to discriminate soil salinity area identification and mapping (Taghizadeh Mehrjardi et al., 2008 and Dwivedi and Sreenivas, 1998). using remote sensing software. Large scale salinity discrimination was not observed for micro level salinity assessment and therefore same study was taken up for developing a tool in .NET software

\section{Study Area (South Nagapattinam district)}

The study was conducted in Nagapattinam district of Tamil Nadu State, India, which is bounded on the north by Cuddalore district, south by Palk Strait, East by Bay of Bengal and west by Thiruvarur district. The area lies between $10^{\circ} 34^{\prime} 32.69^{\prime \prime}$ and $10^{\circ} 46^{\prime} 57.77^{\prime \prime} \mathrm{N}$ latitude and between $79^{\circ} 47^{\prime} 02.29^{\prime \prime}$ and $79^{\circ} 51^{\prime} 38.24 "$ E longitude in figure 1. 


\section{PRINCIPLE OF TOOL DEVELOPMENT}

The principle of the invention is image acquisition, image processing, image transformation techniques, and image classification (Hick and Russell, 1990.).

Satellite Image Salinity Analyzer (SISA) tools makes use Landsat TM image in the form of .jepg file format. The image in georeferenced format is sub setted to the desired area depending on the saline area. Such image is subjected to image processing and then to image classification. Classification methodology is entirely different where the user can make use of (SISA) to derive any number of intervals based on the threshold values of the digital numbers. Such discrimination would certainly hold the farmers and policy planners to adapt to certain technology to choose different crops for different types of salinity level.

\section{METHODOLOGY}

\section{Sisa - Module 1}

This is a specialized simulation tool used to measure accurate saline levels. This software is developed based on GIS (Geographical Information System) to find out the threshold value, area and scale of the images. It is developed using the VB.NET codes especially to find out the white region present in the image. The flow chart of module 1 is provided below.

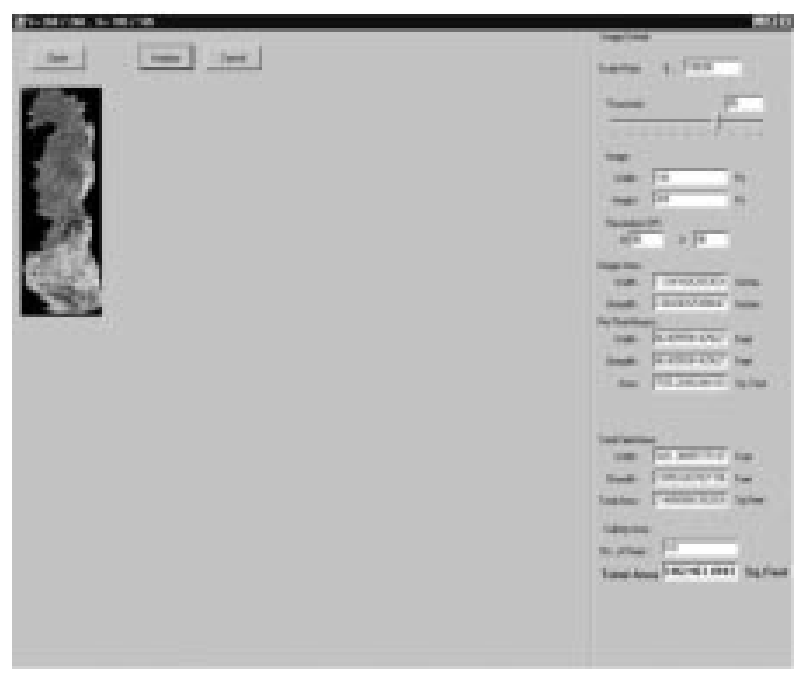

Fig 1. The screenshot of the Module 1

\section{Flow Chart-module 1:}

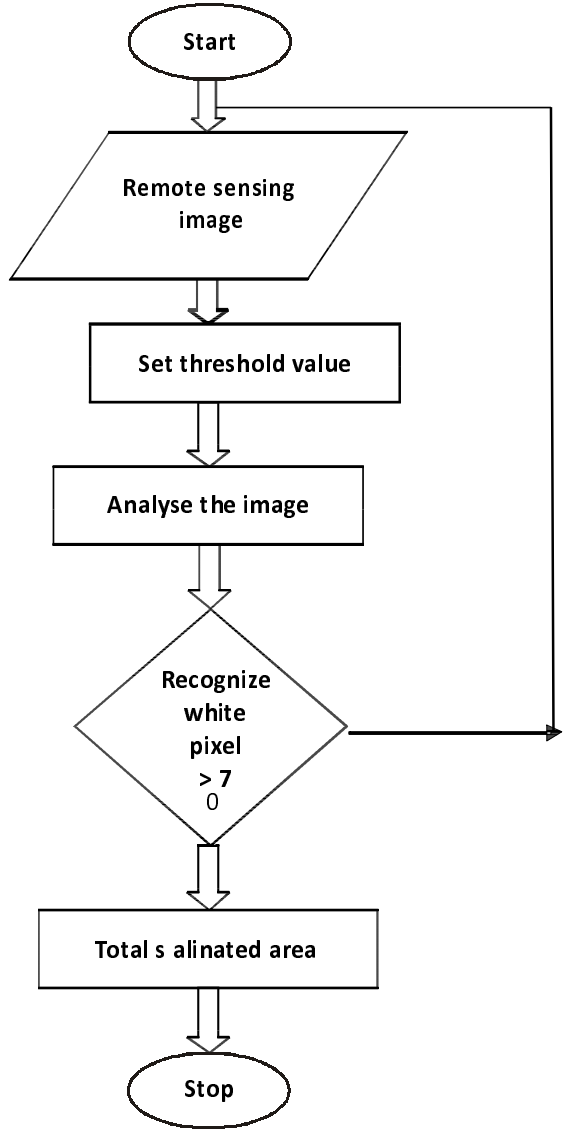

The SISA module1 has been programmed to calculate total salinity present in any area in figure 2 . VB.NET software was used to fine tune the codes. The satellite image in the .jpeg format was given as the input, the image was analyzed and the soil salinity has been calculated for various levels of threshold value and thus the soils are classified according to their threshold value and were set accordingly. Salinity level were validated in the field according to the saline intervals, verification of the field observed values was nearer to the values derived from the SISA method. Thus the white pixels in the image are recognized properly the total salinated area was calculated.

\section{Sisa Module-2}

The various factors responsible of the salinity are given as input to the model and thus the software were made to run below was represented in a block diagram of the module-2. Satellite image salt analyzer was used to find the accurate are mapping data discrimination and stochastically represent them statistically in the form of bar diagrams and graphs. The SISA module classifies the image at three levels as three different 
images of salinity (low, medium \& high) with different colour representation for each class (green, blue, red). This factor was referred as false contouring in this study.

A pictorial representation bar chart in figure 3 represent Low, moderat and high saline areas of Nagapattinam district and a pie chart based on threshold value figures out that percentage of salanisation in Nagapattinam district.

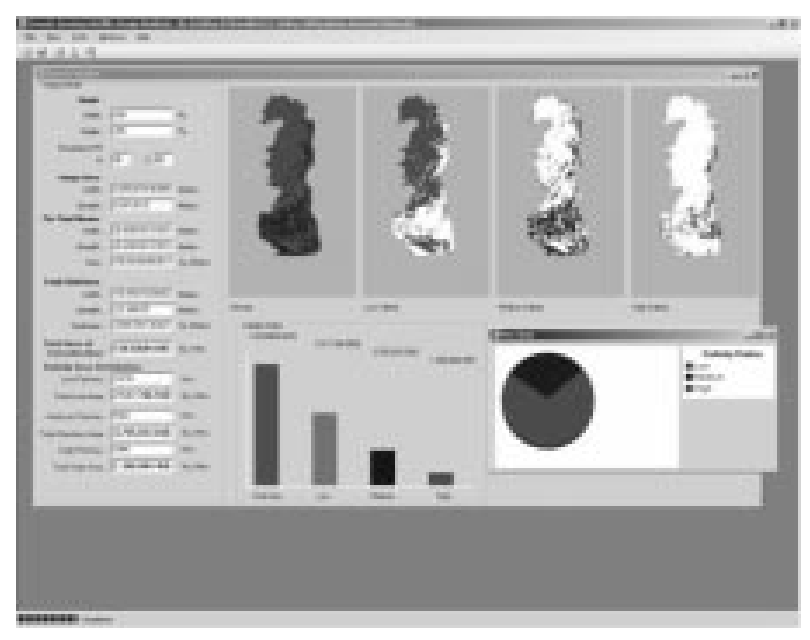

Fig 2. Screenshot of the SISA Module 2.

The SISA module2 has been programmed to calculate three levels of salinity based on the threshold value. The satellite image in the jpeg format was given as the input, the image is analyzed and the three levels of soil salinity has been calculated and thus the soils were classified as low, medium and high salinity areas. And thus the white pixels in the image are recognized as total saline area has been calculated.

\section{RESULTS AND DISCUSSION}

\section{Classification Of Soil Salinity}

The image was classified by using false color and threshold values in a supervised classification algorithm, unsupervised classification iso-data classification and hybrid classification. Finally three different classes were recognized based on the training samples that are as follows

(1) Soils with low salinity $(\mathrm{TH}<50)$,

(2) Soils with medium salinity $(\mathrm{TH}=50-70)$

(3) Soils with high salinity $(\mathrm{TH}>70)$
Flow Chart Module -2
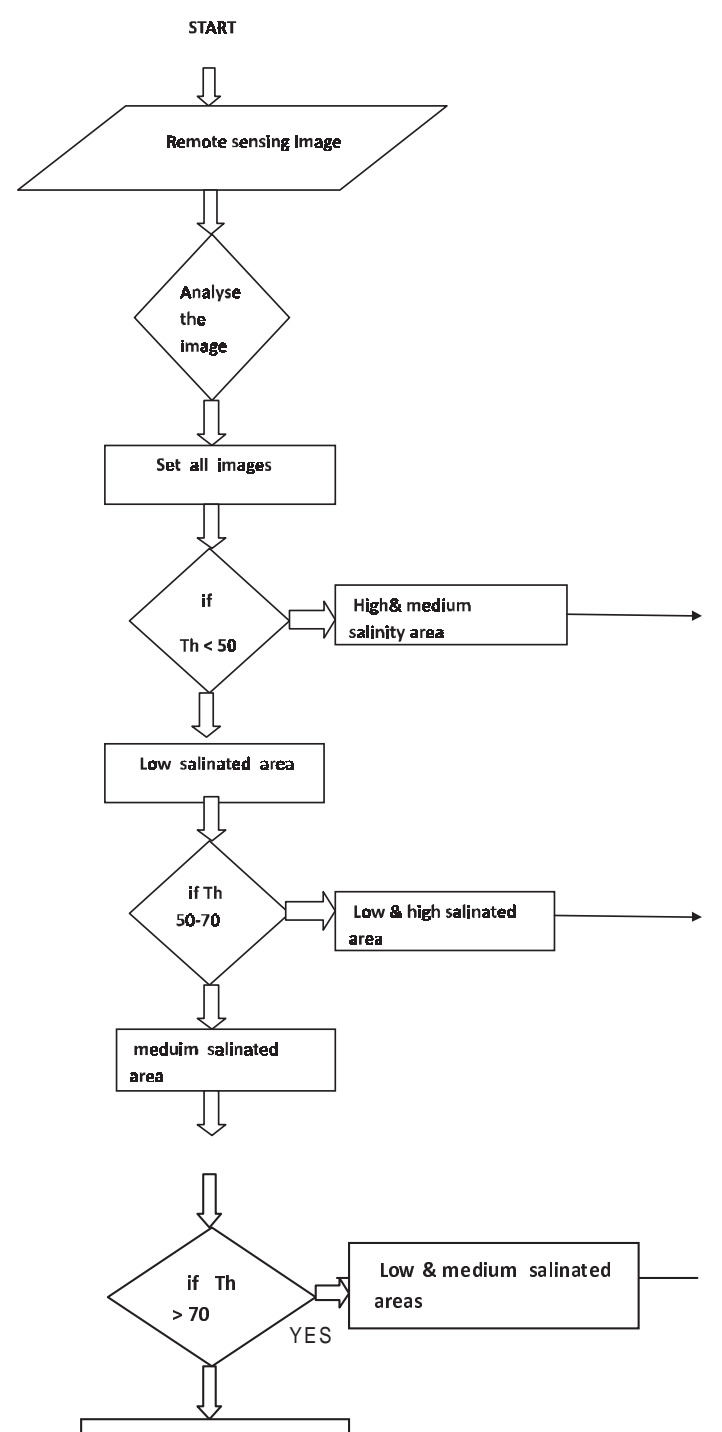

high salinated area

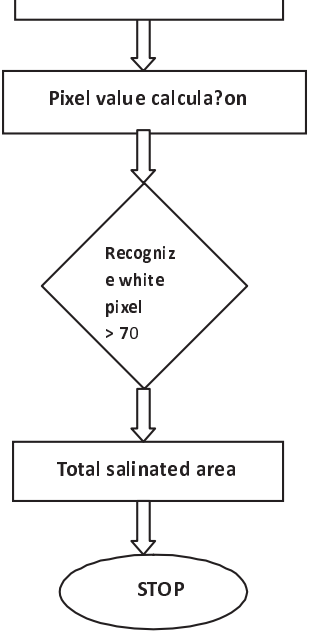




\section{LOW SALINE AREAS}

Based on the threshold value on the satellite image analyzer the low saline areas in the image are been identified. Low salanisation was calculated by the white field mixed with other colors (i.e.) there are traces of white patches scattered over the image where the RGB value of white is nearly 255 . As the threshold value was $<=50$ low saline areas in the image were indicated by green color. In Nagapattinam district, agriculture was practiced in the low saline areas. No visible salts are been identified in the soil surface of these areas. Spatially crops distributions are uneven and patchy. There are not greater impacts of soil saline on the cultivated crops in this region.

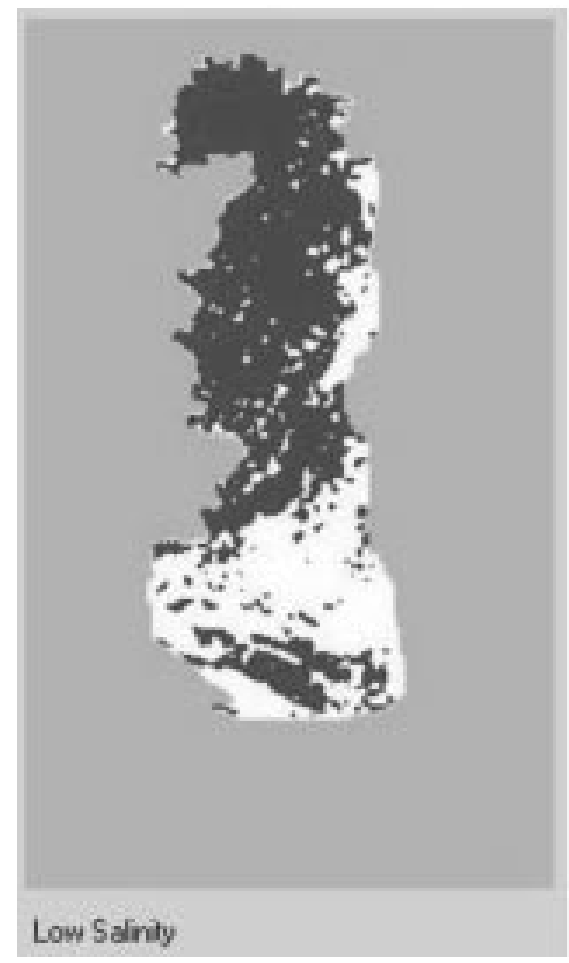

Fig. 3 Low salinity areas

\section{Moderate Saline Areas}

Based on the threshold value on the satellite image analyzer the moderate saline areas in the image have been identified. Moderate salination was calculated by the white field mixed with other colors (i.e.) like traces of white patches scattered over the image where the RGB value of white is nearly 255 and then the threshold value were between 50 and $<=70$. Moderate saline areas in the image are indicated by blue color. Salts distribution are fairly visible on the soil surface on moderately saline areas. Crops were sparsely distributed on these areas. Vegetative growth of this crops were restricted due to moderate salanisation of the soils coupled with saline water due to that reverse osmosis would have occurred on the crops lead to wilting of crops. The soil in these region has been degraded by the moderately scattered salt patches in this region.

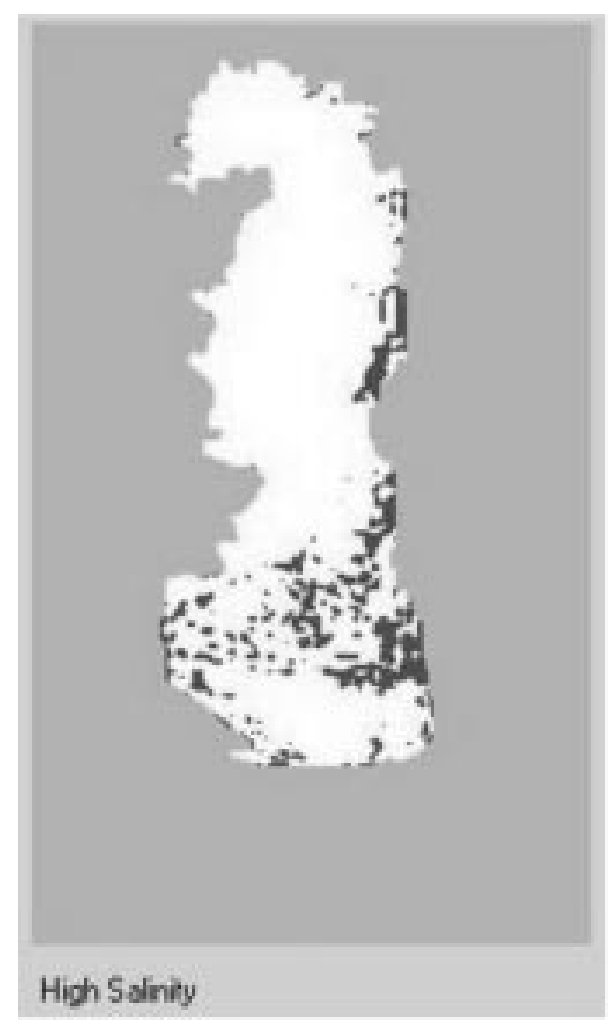

Fig. 4 Medium salinity areas

\section{High Saline Areas}

Based on the threshold value on the satellite image analyzer the high saline areas in the image have been identified. High saline was calculated by the white colors uniformly present in the image (i.e.) the traces of pure white patches scattered over the image where the RGB value of white was exactly 255 and then the threshold value was $\Rightarrow>70$. Such saline areas in the image are indicated by red color. In Nagapattinam, the high saline areas was observed to be 0.345 sq. km. contributing approximately 12 percent of the total study area. Agriculture was strictly restricted as salts are fairly visible on the soil surface of high saline areas. Crop growths was restricted due to higher $\mathrm{pH}$, electrical conductivity that results in crop wilting due to reverse osmosis processes. 
Table 1. Saline classification, threshold value and percent area distribution.

\begin{tabular}{|c|c|c|c|c|}
\hline $\begin{array}{c}\text { Salinity } \\
\text { level }\end{array}$ & $\begin{array}{c}\text { Thresho } \\
\text { Id } \\
\text { value }\end{array}$ & $\begin{array}{c}\text { Area } \\
\text { Sq } \\
\text { Kms }\end{array}$ & $\begin{array}{c}\text { Percent } \\
\text { area\% }\end{array}$ & Remarks \\
\hline LOW & $<50$ & 3.19 & 60.0 & $\begin{array}{c}\text { No visible } \\
\text { salts on the } \\
\text { soil surface }\end{array}$ \\
\hline MODERATE & $\begin{array}{c}50 \\
<=70\end{array}$ & 0.93 & 29.1 & $\begin{array}{c}\text { Salts are } \\
\text { fairly visible }\end{array}$ \\
\hline HIGH & $>70$ & 0.346 & 11.9 & $\begin{array}{c}\text { Soil surface } \\
\text { is fluffy }\end{array}$ \\
\hline
\end{tabular}

Such soil need more remedial measures like leaching, treatment with gypsum, and planting of salt resistant crops for the benefit of farming community (Farifteh al., 2006).

The soil in these regions has been degraded by the highly scattered salt patches in this region (Agbu P.A., 1990).

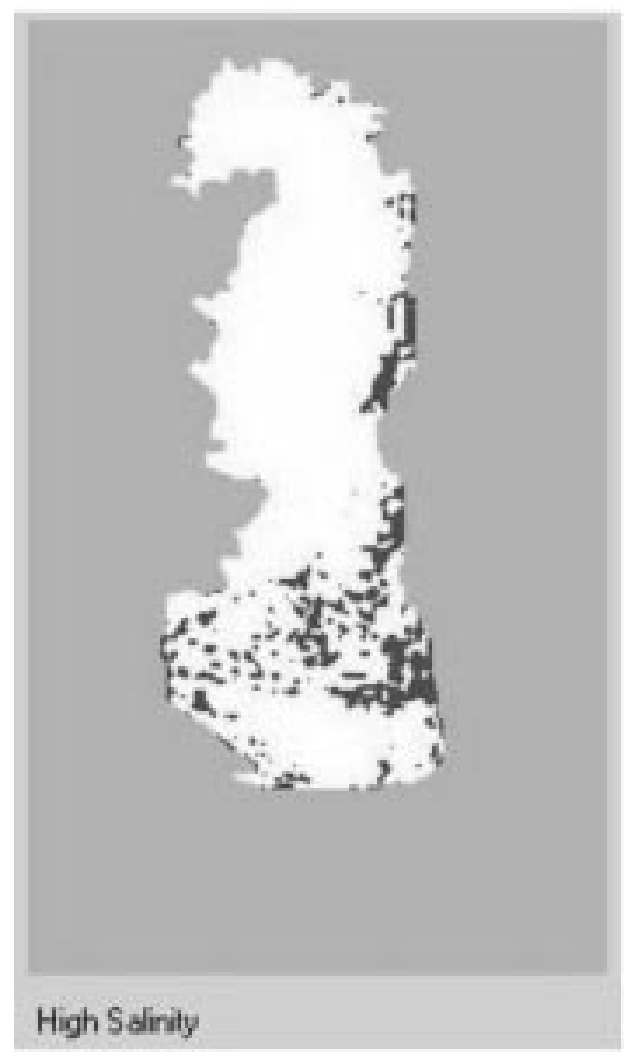

Fig. 5. High salinity areas

\section{CONCLUSION}

The sequence of the model from detection, observations, correlation and verification, and mode validation has proved to be applicable for mapping salinity using SAS and GIS techniques. From the above results it is concluded that the use of remote sensing data followed by site observations is a powerful tool in detecting saline areas. The model shows that most of the saline areas delineated using remote sensing data fits well with those delineated using site observations data, which gave a good indication for the validity of the model. The model can be used in Nagapattinam district areas that experience salinization problems. Thus Southern Nagapattinam of Tamil Nadu has been classified to different classes of saline soils based on its intensity.

\section{REFERENCES}

[1] Agbu P.A., Fehrenbacher D.J. \& Jensen .I.J., 1990. Soil property relationships with SPOT satellite digital data.

[2] Dwivedi.R.S., K. Sreenivas (1998). Delineation of salt-acted soils and Water logged areas in the Indo-Gangetic plains using IRS-1C LISS-IIIdata. International Journal of Remote Sensing, 19:14, 2739 - 2751.

[3] Farifteh,J.,Farshad, A.,\& GeorgeR.J.(2006). Assessing salt-affected soils using Remote Sensing, solute modeling, and geophysics. Geoderma, Volume 30, 3-4, pp. 191-206.

[4] Hick P.T. \& Russell W.G.R., 1990. Some spectral considerations for remote sensing of soil salinity. Australian Journal of Soil Research, 28(3), 417-431.

[5] Taghizadeh Mehrjardi.R,Sh. Mahmoodi,M. Taze E.Sahebjalal. (2008).Accuracy Assessment of Soil Salinity Map in Yazd-Ardakan Plain, Central Iran, Based on Land sat ETM+ Imagery, American-Eurasian J. Agric. \& Environ. Sci., 3 (5):708-712, 2008

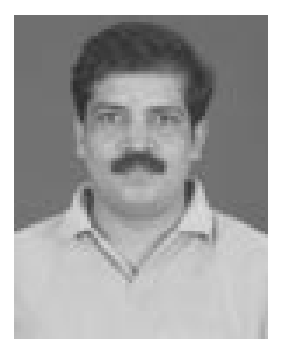

Dr. V. E. Nethaji Mariappan is working as Scientist-D at Centre for Remote Sensing and Geoinformatics at Sathyabama University. He has adequate research experience in the field of Remote Sensing and GIS on Agriculture and Soil. 